\title{
System Level Design as Applied to CMU Wearable Computers
}

\author{
ASIM SMAILAGIC \\ DAN SIEWIOREK
}

\author{
Institute for Complex Engineered Systems, Carnegie Mellon University, Pittsburgh, PA 15213
}

\begin{abstract}
The paper describes a system level design approach to the wearable computers project at Carnegie Mellon University (CMU). The project is an unique example of a cross-disciplinary effort, drawing students from mechanical engineering, electrical and computer engineering, computer science, and industrial design. Over the last six and half years that the course has been taught, teams of undergraduate and graduate students have designed and fabricated sixteen new generations of wearable computers, using an evolving artifact-specific, multidisciplinary design methodology. The complexity of their architectures has increased by a factor of over 200, and the complexity of the application has also increased significantly. We introduce a metric to compare wearable computers and show that their performances have increased by several orders of magnitude. A system-level approach to power / performance optimization is going to be a crucial catalyst for making wearable computers an everyday tool for the general public.
\end{abstract}

\section{Introduction}

Products of the future will be technologically more sophisticated, highly customized, produced in small batches, and brought to market quickly. These products will require an approach which integrates the application, artifacts, the CAD environment, and physical prototyping. These trends are imposing specific requirements on the university engineering education and expertise that graduated students bring to industry. Carnegie Mellon's innovative Wearable Computer Course has been offered over the last six and a half years. The students in this multidisciplinary project course design, assemble and fabricate a new generation of wearable computers for a specific client each term. The course builds upon itself in that a history of the design process, technical decisions and improvements can be maintained for different artifact generation.

Throughout the design of the sixteen generations of wearable computers, an interdisciplinary concurrent design methodology (ICDM) has evolved [1],[2],[3],[4]. As a result of the ICDM methodology, we have achieved a four month design cycle for each new generation of wearable computers. The cycle time of the new products is ideally suited to the academic semester. The goal of the design methodology is to allow as much concurrency as possible in the design process. Concurrency is sought in both time and resources. Time is divided into phases. Activities within a phase proceed in parallel, but are synchronized at phase boundaries.

Since wearable computers represent a new paradigm in computing, there is no consensus on the mechani$\mathrm{cal} / \mathrm{software}$ human computer interface or the capabilities of electronics. Thus iterative design and user evaluation made possible by a rapid design/prototyping methodology is essential for quick definition of this new class of computers. We have chosen to create a course centered on wearable computers because a majority of engineering design projects require the resolution of multi-designer, concurrent, highly-constrained and conflicting constraints.

In the long term, it is clear that a system-level approach to power / performance optimization will be a crucial catalyst for making wearable computers an everyday tool for the general public. Energy efficiency must be considered in all phases of system design.

Other examples of system design projects include: the Infopad project at UC Berkeley [5], the Wireless Modem project at UCLA [6], and the Broadband Wired and Wireless Modem project at IMEC [7]. These projects are system design efforts that include a multi-disciplinary research with an intense interaction with the system industry.

\section{Design Methodology}

The multidisciplinary design evolves in parallel, ensuring a strong crosstalk among the different discipline teams. The collaboration is attained through a framework of compatibility between interdisciplinary design tools and agreement on a design language and representations so that the latest information from each discipline is always available and understandable to the others. To provide flexibility to the decision process during the initial design stages, each discipline formulates the problem from its point of view in terms of design goals instead of design constraints. 
Resources consist of personnel, hardware platforms, and communications. Personnel resources are dynamically allocated to groups which focus on specific problems. Communications allow design groups and individuals to communicate between the synchronization points.

Table 1 depicts the evolution of the ICDM through the first four generations of wearable computers. The first column in Table 1 represents the steps in the product cycle from conception through manufacturing that deal with the artifact. As the methodology evolved through successive generations of more capable wearable systems, more sub-phases were added as to refine the original phases.

\subsection{Conceptual Product.}

During the conceptualization stage, the multidisciplinary design team establishes a common vision of the end product. This vision provides a consistent set of design goals for all disciplines to maintain throughout the product development cycle. Without a common understanding or vision between design groups and their members, each would be forced to rely on their own set of assumptions and criteria based on only a single view of the product. As experience was gained with deployment of wearable computers, the key role of users became apparent.

\subsection{Configurational Design}

During the first two generations, the systems were derived directly from the feasible technology. Subsequently the product configuration became a critical phase. It results in system architecture and subsystem specification.

\subsection{Detailed design.}

The detailed design phase is traditionally defined by a well developed methodology and a rich set of CAD tools. A detailed design of each subsystem is performed, with particular attention to maintaining the interface specifications as defined in the Product Design Specification.

\subsection{Manufacturing}

The manufacturing phase uses a combination of electronic and mechanical job-shops and on-campus rapid prototyping facilities. After detailed design is completed, each group implements their subsystem using the acquired technology. As implementation progresses, the subsystems are demonstrated at various stages of development.

System design and engineering is performed by the class as a whole and then the various disciplines perform detailed design and implementation. During the whole process, the four disciplines interact along well defined design boundaries. The hardware design must merge with the mechanical/industrial engineering design so that the hardware fits within the case and so that adequate power is available for the hardware. The hardware must merge with the software design so that adequate resources are available for the necessary functions of the software and so that software drivers are available for the hardware. The software design must merge with the user interface design so that input/output can be performed and so that the user has available the functions necessary to perform their task. Finally, the user interface design must merge with the mechanical/industrial design to capture the interactions between the system and the user.

\section{Wearable Computers}

Over the past 20 years, there has been steady progress toward making computers more mobile. This has been driven by both higher processor integration levels and the development of high-performance batteries. As information becomes increasingly available through wireless technology and the Internet, conventional mobile platforms (such as laptops) that are heavy and impede mobility quickly become less appealing.

The Wearable Computers project at Carnegie Mellon University is defining the future for not only computing technologies, but also for the sue of computers in daily activities. The goal of this project is to develop a new class of computing systems with a small footprint that can be carried or worn by a human, and be able to interact with computer-augmented environments. By rapid prototyping and fast development of new artifacts and concepts, CMU has established the new paradigm of wearable computers. The sixteen generations of wearable computers have been designed and built over the last six and a half years, and most of them field tested as well. Several of them relevant to this paper are summarized below. The first system was a technology exploration vehicle, seeking lightweight, low-energy solutions to mobile environments with 1) long range and 2) short range, dense infrastructure. The following two systems are pentium-based wearable computers in very small packaging. The next system represents a family of wearable computers dedicated to speech translation applications. The last one is a very low power consumption StrongARM based wearable computer providing high processing capabilities.

- ISAAC (Integrated Speech - Activated Application Control) is a technology feasibility prototype which explores capabilities in a low weight, low energy area, assuming communication over short distances. ISAAC employs speech for communication with the infrastructure and special rooms around campus. A wireless microphone transmits analog speech to a speech recognition system on a base computer. The base computer can control devices through infrared repeaters and communicates with the user via an infrared wireless headset. The user can read electronic mail, or control the presentation (e.g. VCR, slide projector) using audio input/output [8].

- $\quad$ TIA-P is a commercially available system, developed 


\begin{tabular}{|c|c|c|c|c|}
\hline The Product Cycle & VuMan 1 & Vuhan 2 & Navigator & Vuhan 3 \\
\hline \multirow[t]{8}{*}{ Conceptual Product } & - Technology Survey & - Technology Survey & - Technology Survey & - Problem Definition \\
\hline & -mechanical form & - technology assessmen & - technology assessment & - requirements analysis, user \\
\hline & & - feasibility study & - mechanical form & needs evaluation \\
\hline & & (including wire-wrap & - product feature matrix & - product design definition \\
\hline & & prototyping) & & - Technology Survey \\
\hline & & - mechanical form & & - technology assessment \\
\hline & & & & - mechanical form \\
\hline & & & & - product feature matrix \\
\hline \multirow[t]{7}{*}{ Configurational Design } & & & - System Architecture & - System Architecture \\
\hline & & & - Specification & Specification \\
\hline & & & - dependency matrix & - dependency matrix \\
\hline & & & - Subsystem & - Subsystem Specification \\
\hline & & & Specification & - product design \\
\hline & & & - product design & specification \\
\hline & & & specification & \\
\hline \multirow[t]{7}{*}{ Detailed Design } & - Detailed Design & - Detailed Design & - Detailed Design & - Detailed Design \\
\hline & -place and route & -place and route & - place and route & - place and route \\
\hline & -thermal analysis & -protoboard providing & - protoboard providing & - mechanical/electronic/ \\
\hline & & -geometric information & geometric information & software mock-ups \\
\hline & & - thermal analysis & - thermal analysis & - thermal analysis \\
\hline & & & - task dependency graph & - task dependency graph \\
\hline & & & & - user feedback \\
\hline \multirow[t]{11}{*}{ Manufacturing } & - Implementation & - Implementation & - Implementation & - Implementation \\
\hline & - printed circuit boards & - printed circuit boards & - printed circuit boards & - printed circuit boards \\
\hline & - vacuum formed & - rapid prototyping of & - pressure formed housing & - rapid prototyping of \\
\hline & housing & housing & - evolving list of open & housing \\
\hline & - System Assembly & - System Integration & issues & - revolving list of open \\
\hline & - (30 minutes/unit) & - (2 minutes/unit) & - System Integration & issues \\
\hline & & & - integration tree & - System Integration \\
\hline & & & & - integration tree \\
\hline & & & & - test of the working system \\
\hline & & & & - Design Methodology \\
\hline & & & & Evaluation \\
\hline
\end{tabular}

Table 1. Product Cycle Steps and Corresponding Design Methodology Phases

by $\mathrm{CMU}$, incorporating a $133 \mathrm{MHz} 586$ processor, 32MB DRAM, 2 GB IDE Disk, full-duplex sound chip, and spread spectrum radio (2Mbps, $2.4 \mathrm{GHz}$ ) in a ruggedized, hand-held, pen-based system designed to support speech translation applications. TIA-P supports the Multilingual Interview System/Language Translation that has been jointly developed by Dragon Systems and the Naval Aerospace and Operational Medical Institute (NAOMI) [9].

- TIA-0, show in Figure 1, is a small form factor system using the electronics of TIA-P. The entire system including batteries weighs less than three pounds and can be mission-configurable for sparse and no communications infrastructures. A spread-spectrum radio and small electronic disk drive provide communications and storage in the case of sparse communications infrastructure whereas a large disk drive provides self-contained stand-alone operation when there is no communication infrastructure. A full duplex sound chip supports speech recognition. TIA-0 is equivalent to a Pentium workstation in a softball sized packaging. The very sophisticated housing includes an embedded joypad as an alternative input device to speech [9].

- The Smart Modules are a family of wearable computers dedicated to the speech processing application. A smart module provides a service almost instantaneously and is configurable for different applications. The speech recognition module uses CMU's Sphinx 2 continuous, speaker independent system [9]. The speech recognition code was profiled and tuned. Pro- filing was done to identify "hot spots" for hardware and software acceleration and to reduce the required computational and storage resources of software. Input to the module is audio and output is ASCII text. The speech recognition module is augmented with speech synthesis. A combination of the language translation module (LT), and speech recognizer (SR) module, forms a complete stand-alone audio-based interactive dialogue system for speech translation [9].

- The Infocom is a very small mobile device, based on the Compaq Itsy, Figure 2 [10], which is a clockthrottleable StrongARM processor with 8 Mbytes of flash memory, 64 Mbytes of random access memory, 3 serial ports, a gray-scale display, and a touch screen. The processor clock can be varied from $30 \mathrm{MHz}$ up to $200 \mathrm{MHz}$. At the highest clock frequency, the processor is rated at approximately 220 Dhrystone MIPS. The Itsy runs the Linux operating system, weighs about 120 grams, and consumes less than 1 watt. We have ported the Sphinx speech recognition system to the Itsy as well as GeoPlex peer software.

\section{Energy Usage in Wearable Computers}

Most of the power consumed by a mobile device can be divided into three major categories: the computer, the display, and the radio. Since the early 1990s, there has been a rapid reduction in milliWatts/MIPS for electronics forming a new energy-based Moore's Law. From 1990 to 1995 , the power / performance ratio dropped by a factor of ten every 2.5 years, and from 1996 is expected to continue 


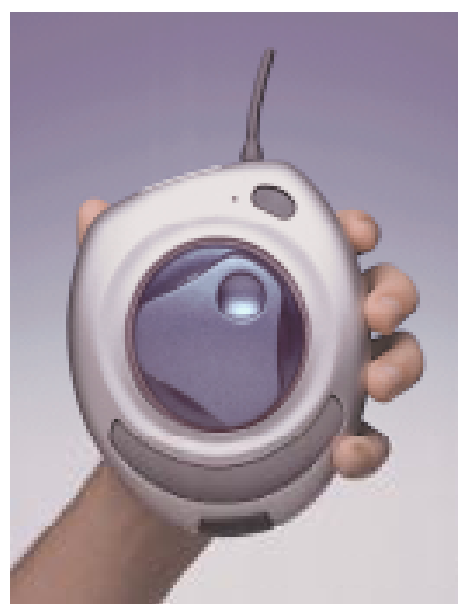

Figure 1. TIA - 0 Wearable Computer

to drop by a factor of four and a half in the next five years [11].

Figure 3 shows that, even with conservative estimates of the rate of improvement in the energy demand of digital logic, CPUs and programmable DSPs will reach performance levels of 1,800 MIPS/W by the year 1999, and as much as 32,000 MIPS/W by 2009. Electronic systems based on this enormous computational capability, along with similar gains in communications systems, could revolutionize building new complex computer systems. Power requirements of programmable digital electronics will follow the trend lines in Figure 3, yielding a power-to-computation ratio of $1 \mathrm{~mW} / \mathrm{MIPS}$ in the year 2001 . To be conservative, it was assumed that interconnect length and other problems expected to arise in moving to deep submicron technology will increase the ratio by a factor of one and a half to a value of $1.5 \mathrm{~mW} / \mathrm{MIPS}$. The trend curves indicate a further reduction by a factor of 128 by the year 2015 to 7.8 $\mu \mathrm{W} / \mathrm{MIPS}$ (a factor of four and a half for every five years). This figure is derated by a factor of ten, yielding a ratio of

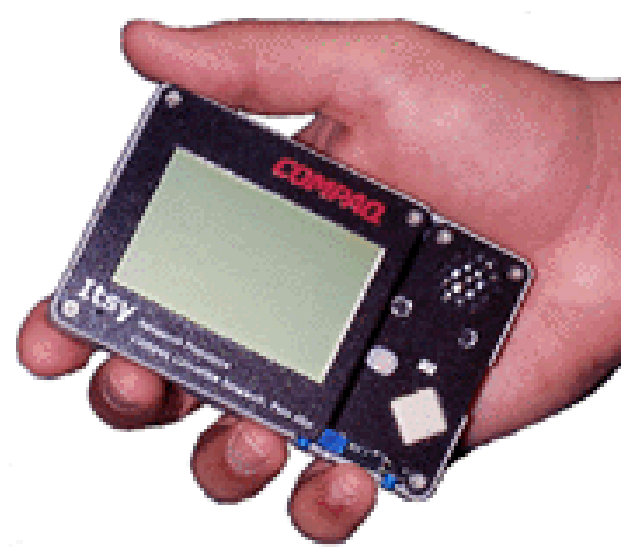

Figure 2. Infocom / Itsy Wearable Computer

$78 \mu \mathrm{W} / \mathrm{MIPS}$ for 2015.

While power consumption of the electronics and the display has been dropping dramatically, power consumption for wireless communication has not kept pace. While a "Moore's Law" for power consumption has emerged, fundamental limitations of radio technology have prevented a similar trend in wireless communications. This suggests that energy consumption of future wearable computers will be communications-bound. For example, the transmit power required to transmit at $16 \mathrm{kbps}$ over $2 \mathrm{~km}$ at $75 \mathrm{MHz}$ is approximately $1.5 \mathrm{~W}$. This power consumption increases as the cube of the distance, and linearly with the data rate, making long-range, high-bandwidth mobile communications prohibitively expensive. In fact, we estimate that by the year 2001 , nearly $80 \%$ of the power consumed by wearable computers will be due to communications.

Table 2 depicts the power consumption of two existing CMU wearable computers of comparable functionality plus a projected system for 2001. As can be seen, while total power drops by over an order of magnitude, the

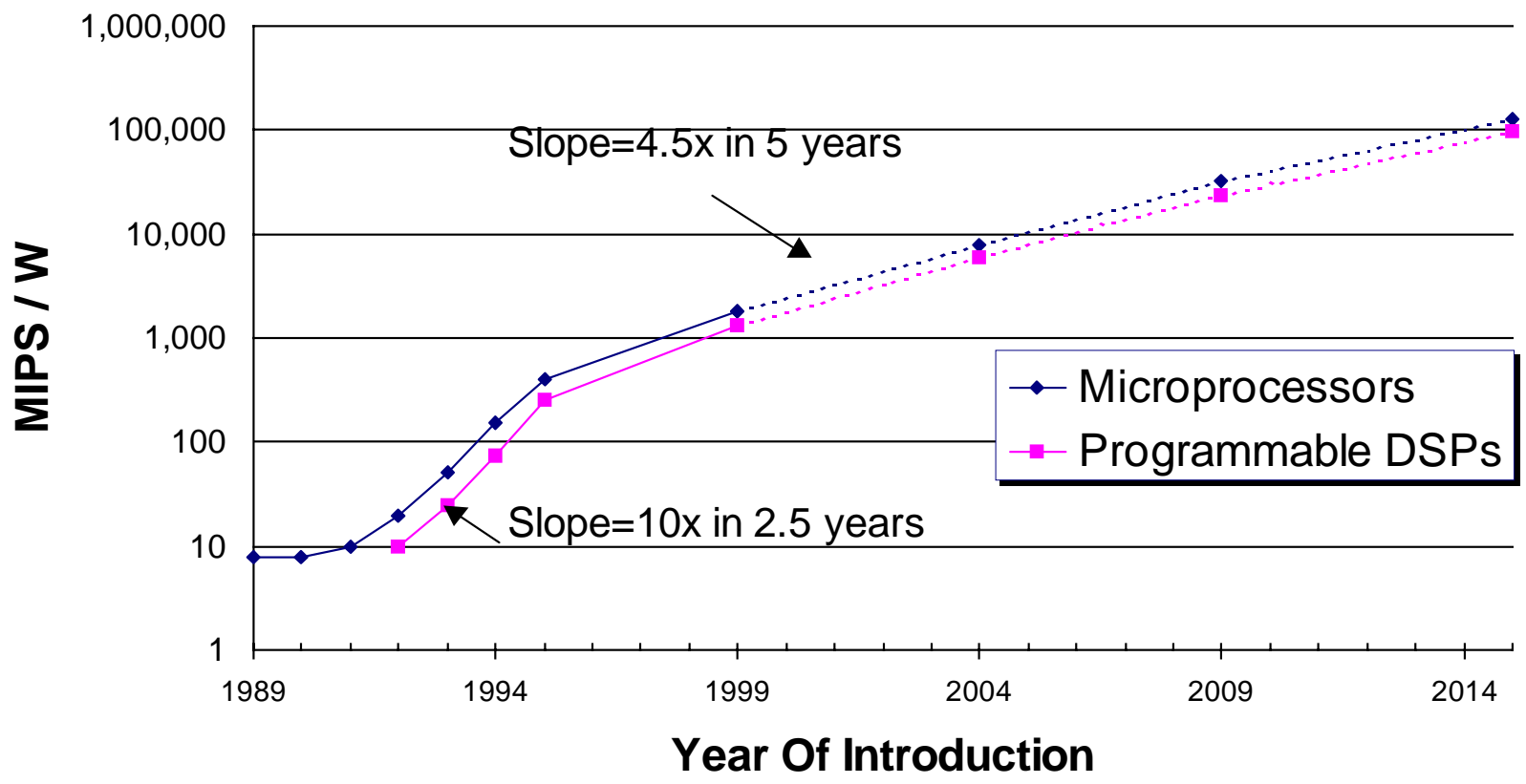

Figure 3. Projected MIPS / W Performance of Microprocessors and Programmable Digital Signal Processors 
relative consumption of power due to radio increases by almost a factor of five.

Energy conservation is one of the key issues in wearable / mobile computing. Battery weight for wearable / mobile computers often exceeds the weight of all other components combined. This makes the systems intrusive and raises environmental concerns due to discarded batteries and battery manufacturing. Furthermore, in some highend portable devices such as cell phones, the cost of battery replacement over two years can exceed the cost of the

\begin{tabular}{|c|c|c|c|}
\hline Fraction of System & 1993 & 1995 & 2001 \\
\hline Computer & $63 \%$ & $29 \%$ & $10 \%$ \\
\hline Display & $19 \%$ & $29 \%$ & $10 \%$ \\
\hline Radio & $18 \%$ & $43 \%$ & $80 \%$ \\
\hline Total Power & $33 \mathrm{~W}$ & $9 \mathrm{~W}$ & $2 \mathrm{~W}$ \\
\hline
\end{tabular}

Table 2. Power Consumption Trends in Wearable Computers

device itself

In the next section, we will list the major factors in a portable electronic system design, and describe power system design as one of them.

\section{Discipline Relationships}

The five major factors in a portable electronic system include functionality, user interface, physical form factor, power, and sensors.

- Functionality. This determines what the system does including services provided. The functionality includes one or more applications as well as communications between applications, other users, and other computing devices.

- User interface. The user interface describes how the user interacts with the functionality provided by the device.

- Physical Form Factor. The physical form factor includes the device's footprint, thickness, weight, materials, and mechanical interfaces. The form factor also includes location upon the body as well as physical interaction while operating the unit.

- Power. Power includes generation as well as consumption. In addition, the by-product of power consumption, heat, must also be managed.

- Sensors. Sensors provide interfaces to the external environment. These include input sensors such as temperature, pressure, chemical, audio, and video. Outputs can include mechanical actuators (e.g., switches, vibrators, etc.) as well as audio and visual.

Figure 4 illustrates how the various disciplines interact. Electronics is primarily focused on functional- ity and power, software focuses on functionality and user interface, industrial design is concerned not only with the user interface, but also the physical form factor, and mechanical engineering is concerned with the physical form factor and the power. All four disciplines are concerned with sensors.

Decisions made in one design discipline affect decisions in another discipline. The impact of the design decisions, especially across disciplines, can be measured if the cost of the design decision can be reduced to a common "currency". In mobile electronic systems that "currency" is power consumption. The following subsection gives examples of design interactions in a discipline as well as how design decisions in each domain can be reduced to energy consumption.

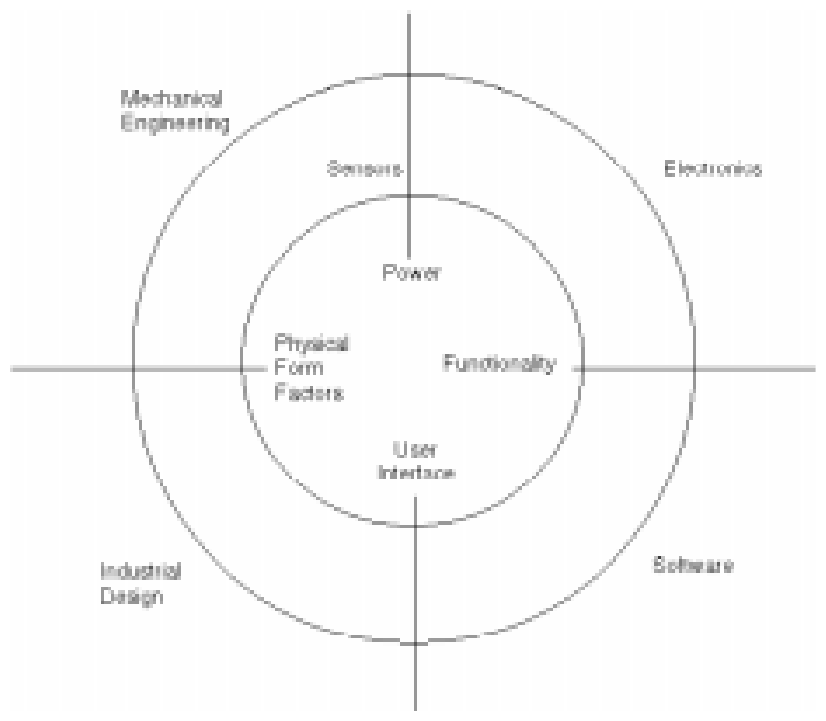

Figure 4. Major Factors in Portable Electronic Systems and their Relationship to the Design Disciplines.

\subsection{Power System Design}

Consider the power system in Figure 5 in which there is collaboration between electronics and mechanical engineers. Power and physical form factor are related by the amount of area required to maintain a comfortable operating temperature for both the electronics and the human.

Temperature $=($ power consumption $) *($ degrees $/$ watt $) /$ (area)

An overview of the power system design process is shown in Figure 5

Requirements generated by the Functionality and the Physical Form Factor provide inputs to the Power System design. In particular, analysis of the functionality produces an operating cycle which is the minimum amount of time that the system should be able to operate completely 


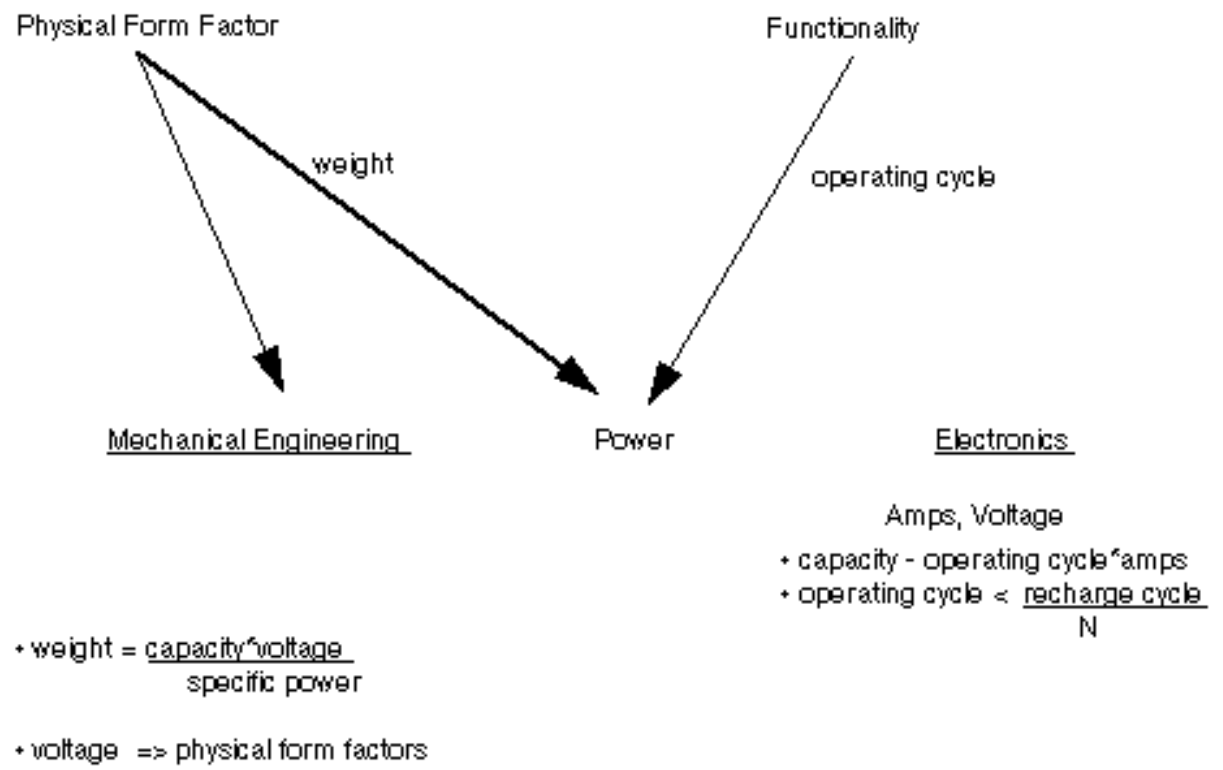

Figure 5. Interactions Between Electronic and Mechanical Engineering in Design of the Power System.

on batteries. The Physical Form Factor produces a weight goal. Frequently the goal is that the battery weight should be less than $50 \%$ of the total weight of the system. The electronics designers use other aspects of the functionality to select electronic components. The total current (measured in amps) of the electronic components required to meet the functionality is estimated. From the operating cycle and the required current the battery capacity can be calculated from the following formula:

$$
\text { capacity }=\text { operating cycle } * \text { amps }
$$

If the batteries are rechargeable, the recharging cycle should be less than the operational cycle. If it is not, $\mathrm{N}$ batteries can be charged in parallel. Thus we have the relationship:

\section{operating cycle $<($ recharge cycle $) / N$}

The variable $\mathrm{N}$ represents the number of extra battery sets required to meet continuous operation broken into durations equal to the operating cycle.

The preliminary design information relating to capacity and voltage is used by the mechanical engineers to determine whether the weight goal can be met. In particular, a battery technology is selected and the weight of the batteries required to meet the functionality is calculated as follows:

$$
\text { weight }=(\text { capacity } * \text { voltage }) /(\text { specific power })
$$

The specific power is a function of the chemical system used in the battery.

The final step is to select the physical form factors of the batteries. The standard form factors usually also provide a standard output voltage. The physical form factors should be selected to provide the voltage that is the dominant voltage required by the electronics. DC-to-DC converters can be used to step down or step up the voltage to meet the requirements of specialty circuits.

If the weight of the battery system exceeds the weight goal from the physical form factor, an alternative battery chemistry can be substituted. If a suitable battery chemistry cannot be found, then the capacity can be reduced by seeking alternative electronics solutions or modifying the energy usage profile of the application during the operating cycle.

\subsection{Examples}

The Navigator 2 wearable computer was intended for sheet metal inspection of aircraft. The inspection requires over 30 hours of close examination of all portions of an aircraft's skin looking for corrosion, dents, cracks, etc. The inspector would maneuver a hydraulic platform to the portion of the aircraft to be inspected and at times would leave the platform to walk or crawl on the aircraft fuselage or wings. Thus hands-free operation of the wearable computer was a very desirable function. Speech recognition became the primary input mode. In addition, the minimal operating cycle was determined to be two hours (i.e., the time between breaks such as lunch or coffee breaks) with a desirable operating cycle of an entire shift or eight hours. Furthermore, the goal was to have no more than one pound of batteries. Figure 6 shows the impact of power management techniques on the Navigator 2. Figure 7 depicts the major components for an all-software solution to speech recognition. A high-performance processor, consuming 6 


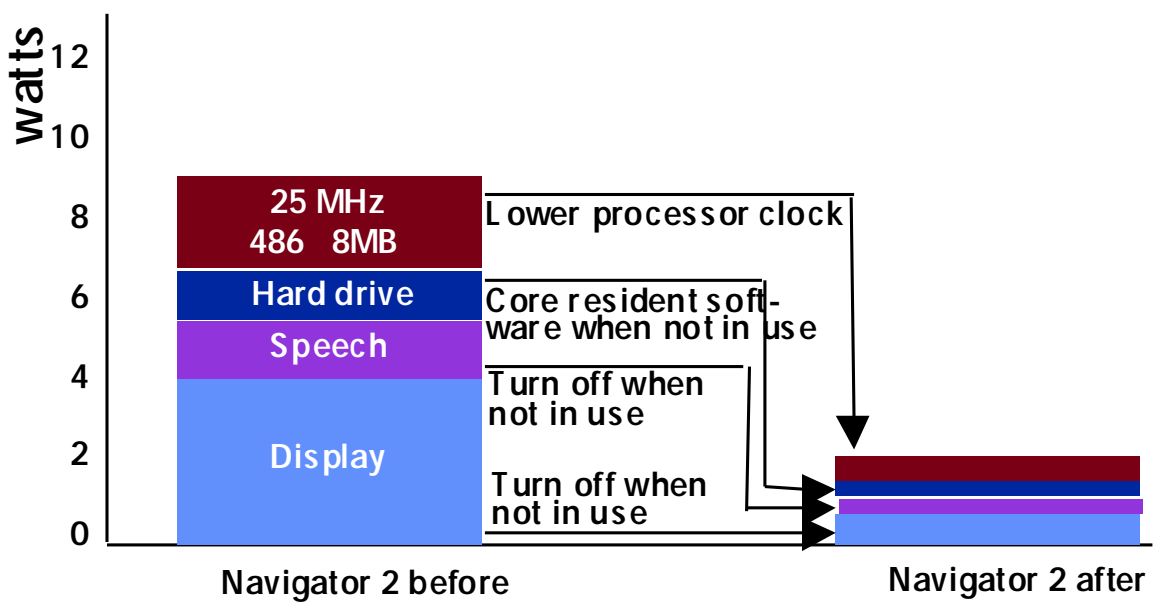

Figure 6. Impact of Power Management on Navigator 2 Wearable Computer

$$
\begin{aligned}
& \text { Software Speech Recognition Solution } \\
& \text { Capacity }=2 \text { hours } * 2.4 \text { amps }+4.8 \mathrm{a}-\mathrm{hr} \\
& \text { Weight }=4.8 \mathrm{a}-\mathrm{h} * 5 \text { volts }=0.53 \mathrm{Kgm} \\
& 45 \mathrm{Wh} / \mathrm{Kgm}
\end{aligned}
$$

$\begin{array}{ll}\text { Processor } & 6 \mathrm{~W} \\ \text { Disk } & 2 \mathrm{~W} \\ \text { Display } & 4 \mathrm{~W} \\ \text { Total } & 12 \mathrm{~W}\end{array}$

Figure 7. Battery Computation for an all Software Speech Recognition System.

watts of power, was required to have enough performance to recognize speech in real-time.

A disk drive and a display added 2 watts and 4 watts respectively for a total estimate by the electronics group of 12 watts. The electronics was operating at 5 volts, yielding a requirement for 2.4 amps. Thus the capacity of the batteries would have to be 4.8 amp-hours. For Nickel Cadmium batteries with a specific power of 45 watt-hours/ kilogram yielded a weight of almost 1.2 pounds.

The electronics group sought alternative solutions and found a dedicated Digital Signal Processor (DSP) that could perform the discrete word speech recognition while consuming only 1.5 watts. The main processor could now be down-graded in performance since the main task, that of speech recognition, was no longer required. A 1.5 watt processor with adequate performance was specified. Fig-

\begin{tabular}{|c|c|c|}
\hline Process & & $1.5 \mathrm{~W}$ \\
\hline Hardwa & & 1.5.W \\
\hline Disk & & $2 \mathrm{~W}$ \\
\hline Display & $4 \mathrm{~W}$ & \\
\hline Total & $9 \mathrm{~W}$ & \\
\hline $9 \mathrm{~W} / 5 \mathrm{ve}$ & 8 am & \\
\hline Capacit! & urs*1 & $\mathrm{Imps}=3.6 \mathrm{a}-\mathrm{hr}$ \\
\hline Weight = & $\begin{array}{r}h r * 5 \\
4.5\end{array}$ & $\begin{array}{l}\mathrm{s}=0.4 \mathrm{Kgm} \\
\mathrm{Kgm}\end{array}$ \\
\hline
\end{tabular}
ure 8 illustrates the computation for the new set of electronics. The resultant weight was less than 0.9 pounds.

The next design decision was the form factor for

\section{Hardware Speech Recognition Solution}

Figure 8. Battery Computation for a DSP Speech Recognition System.

the batteries. The display required 7.2 volts for some of its functions. Many battery cells have a 1.5 volt rating. Since 5 volts was already required for the electronics, thereby requiring a four-battery stack, a fifth battery was added to the stack to have a peak output voltage of 7.5 volts. The next decision was the size of the battery. AA NiCd batteries have a capacity of 1.2 amp-hours. Three stacks of AA batteries would have been required to meet the capacity of 3.6 amp-hours. C cells have a capacity of 1.8 amp-hours, thus the final decision was to go with two stacks of five $\mathrm{C}$ cells each.

Once the system was functioning correctly, a series of energy management techniques was employed to cut the power consumption by a factor of four.

- Lowering Processor Clock Frequency. The processor clock was lowered to the minimum frequency that provided adequate response for the application. Rather than seeking a software speech recognition solution that would have required at least 100 MIPS, a separate 
dedicated speech application PCMCIA card allowed the selection of a more energy-efficient, lower performance processor. In addition, the processor entered a "sleep" mode when there were no inputs that required processing.

- Hard Disk Drive. The software was modified to be memory-resident to avoid paging from the hard disk drive. Thus the hard disk drive could be disabled except for program loading and data base updates.

- Speech. A separate onset of speech recognition circuit enabled the speech recognition PCMCIA card when there was actual speech to be recognized. Otherwise the speech recognition card was disabled.

- Display. The display was disabled when it was moved from in front of the eyes of the user or after a period of user inactivity.

\section{Impact of User Interface on Energy Consumption}

Now consider the interaction between usage and the design of the user interface. Various forms of user interfaces place varying requirements on the performance an capacity of the electronics. The number of operations to perform a user input or output can be related to energy consumption as:

\section{Energy to do user task =}

(number of functions to perform a user task)

$x$ (millions of operations / function)

$x$ (watts / million operations)

$x$ (time of operation)

The type of data to be exchanged must also be selected. For example, consider filing a report consisting of the answers to 100 different questions each having a single word response selected from a menu. Four possible data representations are:

- Text. Assuming one word per question, an average of five characters per word and 8 buts per character, 4000 bits of information would be generated.

- Audio. Assume that the user files an audio report that requires 60 seconds to complete. Sample encoding for audio requires 2.4 Kbits per second.

- Still picture. Assume a VGA picture composed of $640 \times 480$ pixels with 16 levels of gray scale for black and white. The result is 1.23 million bits of data. A color picture with 8 bits for each primary color requires six times more data, or 7.38 million bits.

- Video. The report could also be filed with video clips. Assuming the VGA quality as the still picture at 30 frames per second, a 10-second video clip requires 300 times more data than the corresponding black and white or color picture. The software designer can reduce the number of bits that need to be transmitted by applying compression algorithms. A video frame can be compressed by a factor of 30 on average and at the expense of eight million operations.

Consider the design of the input interface using variations of user interfaces and data representations.

Table 3 shows the energy required for the computation and communication portions and the battery weight (i.e. a metric which users can more directly relate to from personal experience than the seemingly more abstract Watt-hours) required to perform the task [12]. As can be seen the type of user interface and the type of data selected can have a dramatic impact on the energy consumption and weight of the system. For example, a ten second color video clip without compression would require 37.5 grams of battery weight. As shown in Table 3, the type of interface and type of data can have up to four orders of magnitude difference in energy consumed.

\section{Experiments and Results}

We introduce a metric for comparison of wearable computers, which is proportional to the processing power

\begin{tabular}{|c|c|c|c|c|c|c|c|c|c|}
\hline \multirow[t]{2}{*}{$\begin{array}{l}\text { Phwsical } \\
\text { Interface }\end{array}$} & \multicolumn{2}{|c|}{$\begin{array}{c}\text { User } \\
\text { Interface }\end{array}$} & \multicolumn{3}{|c|}{ Computing } & \multicolumn{2}{|c|}{ Transmit } & \multirow{2}{*}{$\frac{\text { Total }}{\text { Energy }}$} & \multirow{2}{*}{$\frac{\frac{\text { Battery }}{\text { Weight }}}{\text { (kg) }}$} \\
\hline & Type & Data Type & $\begin{array}{c}\text { Inter } \\
\text { face } \\
\text { (mop) }\end{array}$ & $\begin{array}{c}\text { Compre } \\
\text {-ssion } \\
\text { (mop) }\end{array}$ & $\begin{array}{l}\text { Energy } \\
\text { (whr) }\end{array}$ & Bits & $\begin{array}{l}\text { Energy } \\
\text { (whr) }\end{array}$ & & \\
\hline \multirow{5}{*}{ Mechanical } & \multirow{5}{*}{$\begin{array}{l}\text { Textural } \\
\text { (IMIPS) }\end{array}$} & $\begin{array}{l}100 \text { Words } \\
\text { Text }\end{array}$ & 300 & & $8.3 \times 10^{-4}$ & $4 \times 10^{3}$ & $1.4 \times 10^{-5}$ & $8.5 \times 10^{-4}$ & $4.2 \times 10^{-6}$ \\
\hline & & 60 sec sound & 60 & & $1.7 \times 10^{-3}$ & $1.4 \times 10^{5}$ & $4.9 \times 10^{-1}$ & $6.6 \times 10^{-4}$ & $3.3 \times 10^{6}$ \\
\hline & & B\&W still & 30 & & $8.3 \times 10^{5}$ & $1.2 \times 10^{6}$ & $4.3 \times 10^{-3}$ & $4.4 \times 10^{-3}$ & $2.2 \times 10^{-5}$ \\
\hline & & $\begin{array}{c}10 \text { sec color } \\
\text { video }\end{array}$ & 30 & & $8.3 \times 10^{5}$ & $2.2 \times 10^{9}$ & 7.53 & 7.53 & $3.8 \times 10^{-2}$ \\
\hline & & $\begin{array}{c}10 \text { sec color } \\
\text { video }\end{array}$ & 30 & 80 & $6.8 \times 10^{3}$ & $7.4 \times 10^{7}$ & 0.251 & 0.258 & $1.3 \times 10^{-3}$ \\
\hline \multirow{2}{*}{ Audio } & \multirow{2}{*}{$\begin{array}{c}\text { Speech } \\
\text { Recogni } \\
\text {-tion } \\
\text { (150 MIPS) }\end{array}$} & $\begin{array}{c}100 \text { Words } \\
\text { Text }\end{array}$ & 45000 & & 0.125 & $4 \times 10^{3}$ & $1.4 \times 10^{-5}$ & 0.125 & $6.3 \times 10^{-1}$ \\
\hline & & $\begin{array}{l}10 \text { sec color } \\
\text { video }\end{array}$ & 4500 & 80 & $1.9 \times 10^{-2}$ & $7.4 \times 10^{7}$ & 0.251 & 0.27 & $1.4 \times 10^{3}$ \\
\hline
\end{tabular}

Table 3. Interactions Between User Interface and Data Types Upon Energy Required for Computing and Data Transmission 
(SpecInt), representing performance, and inversely proportional to the product of volume, weight, and power consumption (R), representing resource metrics. It shows the normalized performance scaled by volume, weight, and power consumption. Figure 9 was constructed based on the data shown in Table 4. A TI 6030 laptop is taken as a baseline for comparison, and its associated value is one. TIA-0 is a factor of 44 better than the laptop while SR Smart Module is over 355 times better than the laptop (i.e., at least a factor of five better in each dimension). Therefore there are orders of magnitude improvement in performance as we proceed from more general purpose to more special purpose wearable computers. It can be seen that functional specification can yield over two orders of magnitude improvement in composite weight, volume, power, and performance.

The inclusion of the StrongArm based Infocom / Itsy wearable computer brings a significant improvement in performances - close to five orders of magnitude better than a laptop, Figure 10 (9.28 $\mathrm{in}^{3}$ in volume, weighs $3 \mathrm{oz}$., 1.4 Watts power consumption'. Figure 11 presents a comparison of speech recognition wearable computers (TIA-P, TIA-0, SR SM, ISAAC) in respect to application functionality, power consumption, and communication throughput. All results are displayed relative to the TIA-P system. Functionality determines what the system performs, includ-

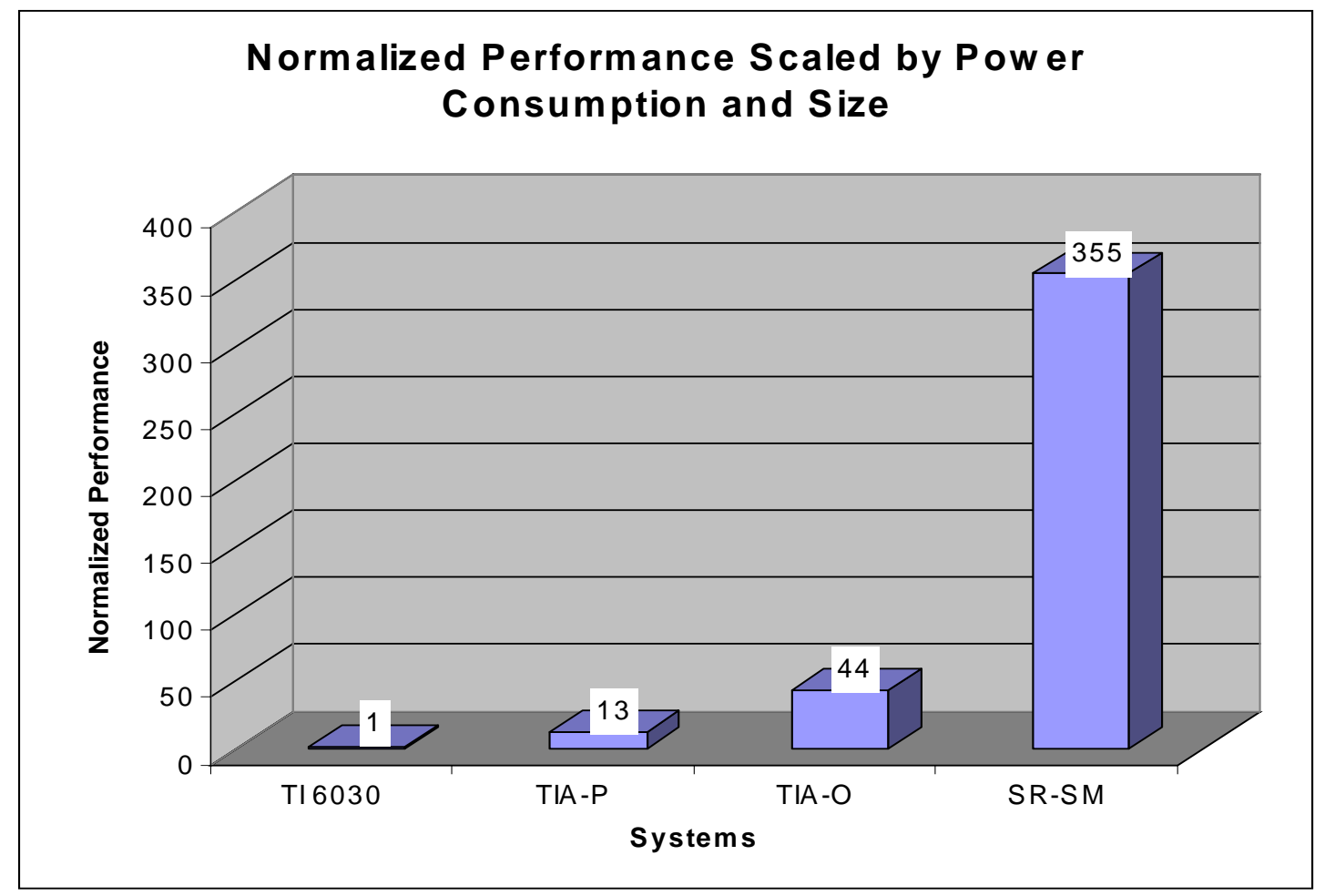

Figure 9. Composite Performance of Speech Recognition Computer Systems

\begin{tabular}{|l|l|l|l|l|l|l|l|l|}
\hline Name & SpecInt & $\begin{array}{c}\text { Volume } \\
(\mathrm{in} 3)\end{array}$ & $\begin{array}{c}\text { Weight } \\
(\mathrm{lbs})\end{array}$ & $\begin{array}{c}\text { Power } \\
(\text { watts })\end{array}$ & $\begin{array}{c}\mathrm{R} \\
\left(\mathrm{V}^{*} \mathrm{~W}-\right. \\
* \mathrm{P})\end{array}$ & SpecInt/R & - Log[SpecInt/R] & $\begin{array}{c}\text { Normalized - } \\
\text { SpecInt/R }\end{array}$ \\
\hline TI 6030 & 175 & 260 & 7.5 & 36 & 70200 & 0.002 & 2.603 & 1.000 \\
\hline TIA-P & 55 & 88 & 3 & 6.5 & 1716 & 0.032 & 1.494 & 12.857 \\
\hline TIA-O & 55 & 45 & 2.5 & 4.5 & 506.25 & 0.109 & 0.964 & 43.581 \\
\hline SR-SM & 175 & 33 & 1.5 & 4 & 198 & 0.884 & 0.054 & 354.545 \\
\hline
\end{tabular}

Table 4. Performance Values Measured and Calculated for Wearable Computers 


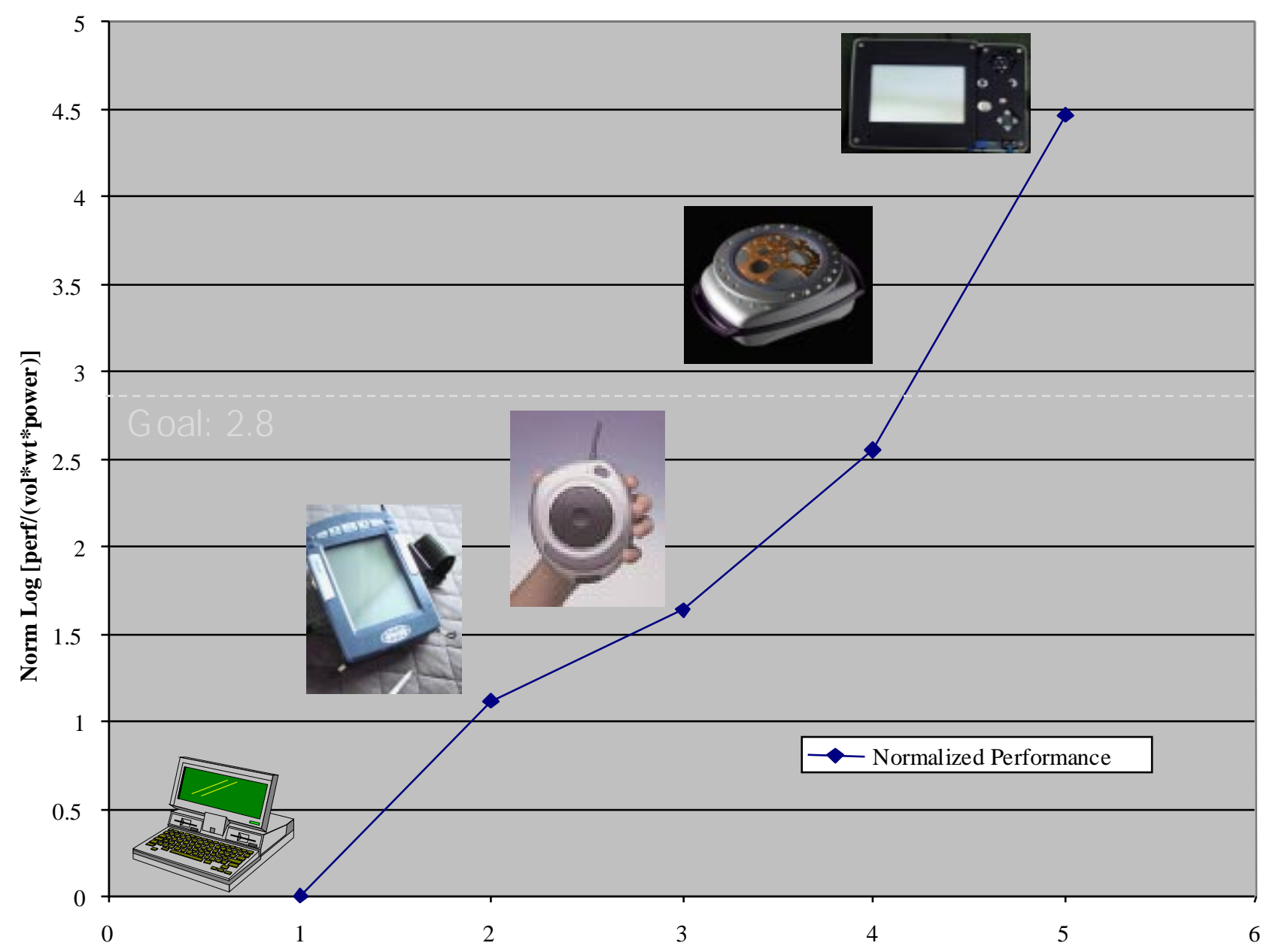

Figure 10. Composite Performance of Speech / Translation Systems

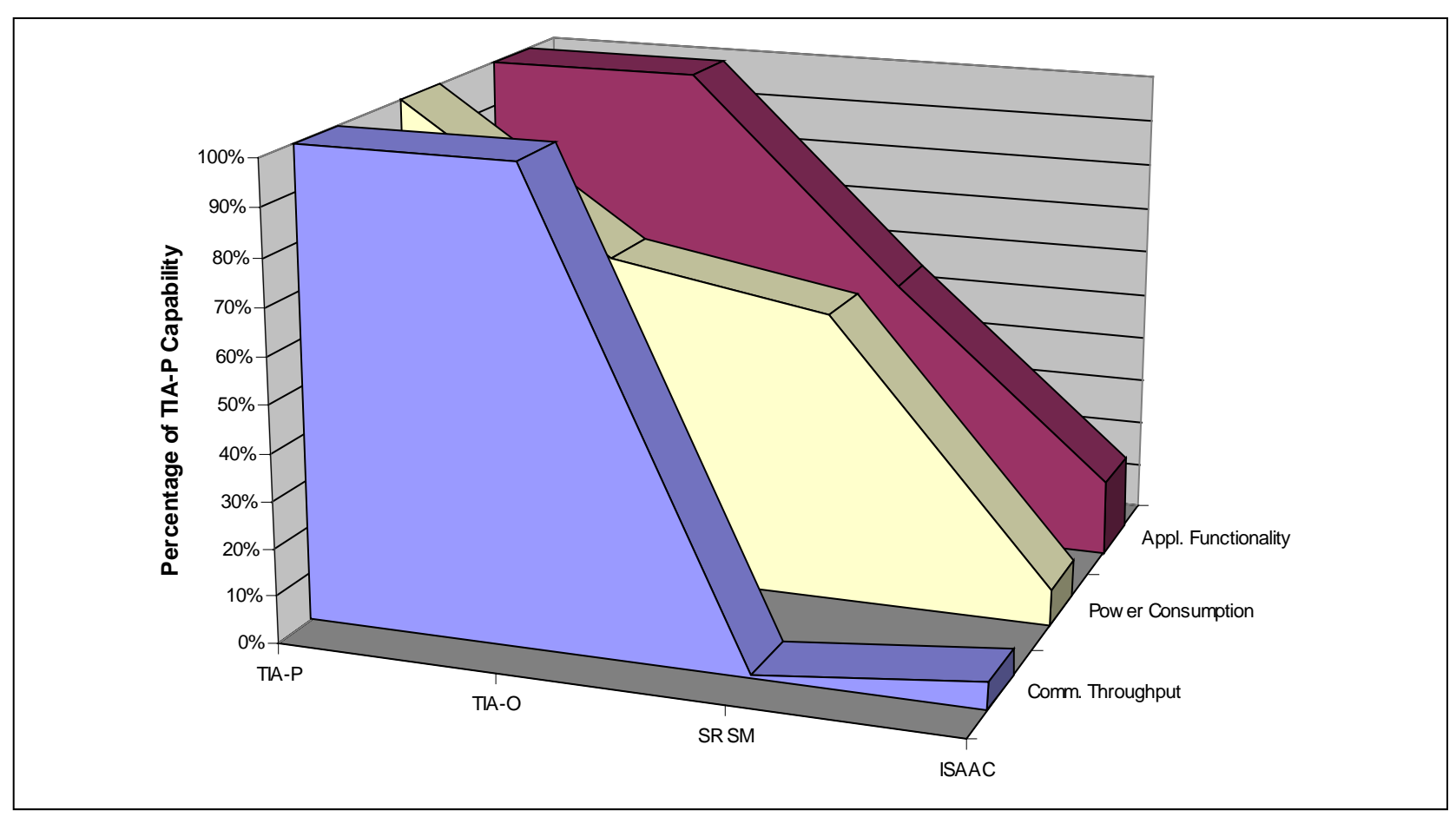

Figure 11. System Comparison Relative to TIA-P 
ing services provided. The functionality graph indicates a system's ability to handle audio, text, diagrams, photographs, and full motion digital video. The diagram shows a continuous trade-off between functionality and resources consumed. General purpose speech recognition computers (TIA-P, TIA-0) provide the highest functionality, but their power consumption is also high. For example, relative to the TIA-P power consumption, TIA-0 consumes $69 \%$, Smart Module 61\%, and ISAAC 7.7\%.

\section{Conclusions}

The wearable computers project at CMU exemplifies the importance of system level design as a new research theme. In the project we not only create new products but also address issues of curriculum development, product innovation, and design methodology. This process fits well in an academic environment, assuming that an adequate laboratory support, CAD tools, and board level fabrication facilities are provided.

The complexity of our wearable computer systems has increased by a factor of over 200, and the complexity of the application has also increased significantly.

A metric for comparison of wearable computers has been introduced, indicating almost five orders of magnitude improvement in performances of our wearable computers running speech recognition applications. Our system-level approach to power / performance optimization addresses important design issues and provides a guidance towards achieving very efficient designs.

\section{References}

[1] D. Siewiorek, A. Smailagic, J.Lee, “An Interdisciplinary Concurrent Design Methodology as Applied to the Navigator Wearable Computer System", Journal of Computer and Software Engineering, Vol.2, No.3, Nov 1994, pp. 259292.

[2] A. Smailagic, D. Siewiorek, et. al., "Benchmarking an Interdisciplinary Concurrent Design Methodology for Electronic/Mechanical Design", Proc. ACM/IEEE Design Automation Conference, San Francisco CA, June 1995, pp. 514-519.

[3] A. Smailagic, D. Siewiorek, et.al. "Very Rapid Prototyping of Wearable Computers: A Case Study of Custom versus Off-the-Shelf Design Methodologies", Proc. ACM/IEEE Design Automation Conference, Anaheim CA, June 1997, pp. 315-320.

[4] C. Amon, A. Smailagic, et.al. "Concurrent Design and Analysis of the Navigator Wearable Computer System: The Thermal Perspective", IEEE Transactions on Components, Hybrids, and Manufacturing Technology, Vol.18, No.3, Sep 1995, pp. 567-577.

[5] R. Brodersen : "Infopad - An Experiment in System Level Design and Intefration", Proc. 1997 DAC, June 1997, pp. 313-314.

[6] C. Chien et. al., "An Integrated Testbed for wireless multimedia computing," Journal of VLSI Signal Processing, Vol. 13, 1996, pp. 105-124.

[7] H. De Man, "Future Systems-On-A-Chip: Impact of Engineering Education," Proc. IEEE Computer Society Workshop on VLSI 1998, Orlando, Fl., April 1998, pp 7883

[8] A. Smailagic, "ISAAC: A Voice-Activated Speech Response System for Wearable Computers", Digest of Papers, First International Symposium on Wearable Computing, IEEE Computer Society Press,October, 1997, pp 183-184. [9] A. Smailagic, "An Evaluation of Audio- Centric CMU Wearable Computers," ACM Journal on Mobile Networking and Applications, 1998

[10] Pocket Computing, http://www.research.digital.com/ wrl/itsy/, C ompaq Western Research Lab

[11] National Research Council, "Energy-Efficient Technologies for the Dismounted Soldier", National Academy Press, Washington, D.C., 1997

[12] D. Siewiorek, "Energy Trade-Offs in Wearable Computer Design," Institute for Complex Engineering Systems Technical Report, Carnegie Mellon University, 1998

\section{Acknowledgments}

The research reported in this paper is supported by the DARPA, NSF Grant on Mobile Computing, and the Institute for Complex Engineered Systems at Carnegie Mellon University. 